\title{
Integrating microvascular assessments into one clinic, in an annual one-stop approach
}

\begin{abstract}
Background: Uncontrolled diabetes can cause many microvascular complications. Thus, early detection and appropriate treatment are essential to prevent diabetes complications that may cause disability and death. The main aim of the study is to test the effectiveness of a one stop screening clinic for retinopathy, nephropathy, and neuropathy for people with diabetes.
\end{abstract}

Methods: A cross-sectional observational study, the study was done during a period of 3 months from February to March 2019. We used convenience sampling to select participants who attended the screening clinic of the Diabetes Care Center at King Salman Hospital, Riyadh, Saudi Arabia. A total of 260 diabetic patients participated in the study.

Results: The study included 260 participants, around $61 \%$ were female participants. The mean age of the participants was 51 years. Most of the participants were type 2 diabetic patients (93.5\%). Patients' acceptance and satisfaction rates of the one stop clinic were $100 \%$. Non-proliferative diabetic retinopathy prevalence was $11 \%$. Meanwhile, the prevalence of maculopathy was $1.5 \%$. The prevalence of micro-albuminuria was $18.6 \%$ and macro-albuminuria was $1.9 \%$. Also, the prevalence of chronic kidney disease stage 3 was $4.2 \%$ and stage 4 was $0.4 \%$. We found that neuropathic symptoms were present in $40.7 \%$ of the participants. The prevalence of diabetic peripheral neuropathy (DNP) according to the neuropathy disability score, which is our gold standard test was $13.8 \%$ and using $10-\mathrm{g}$ monofilament test was $19.5 \%$. Meanwhile, the prevalence of DNP according to DPN-check was $40.9 \%$, and according to Sudoscan was $73 \%$.

Conclusion: Having one clinic that combines retinopathy, nephropathy, and neuropathy screening is possible. A one stop clinic is also highly accepted, reduces clinical visits, and can detect microvascular disease.

Keywords: diabetic retinopathy, diabetic nephropathy, diabetic neuropathy, screening, microvascular complications, diabetes mellitus, Saudi Arabia
Volume 7 Issue 3 - 2020

\author{
Eman Sheshah,' Dalal Al-Qaisi,' Reem Aman,' \\ Ghassan Aldekhayel,' Jumana Alzayer, ${ }^{2}$ Faisal \\ Alerwy, ${ }^{2}$ Abdulrahman Alhadhif, ${ }^{2}$ Maram \\ Alsuwaidan, ${ }^{2}$ Weam Ahmed, ${ }^{2}$ Ahmed Tabash, ${ }^{2}$ \\ Mohammed Alshanqiti ${ }^{2}$ \\ 'Diabetes Care Center, King Salman Hospital, Saudi Arabia \\ ${ }^{2}$ Internal Medicine Department, King Salman Hospital, Saudi \\ Arabia
}

Correspondence: Ghassan Aldekhayel, Diabetes Care Center, King Salman Hospital, Riyadh, Saudi Arabia, Tel 00966555442808 , Email dr.gass@hotmail.com

Received:September 04, 2020 | Published: September 28, 2020
Abbreviations: DM, diabetes mellitus; IDF, international diabetes federation; MENA, middle east and north Africa; ESRD, endstage renal disease; WHO, world health organization; DKD, diabetic kidney disease; CKD, chronic kidney disease; GFR, glomerular filtration rate; ICO, international council of ophthalmology; ADA, American diabetes association; NSS, neuropathy symptom score; NDS, neuropathy disability score; DPN, diabetic peripheral neuropathy; NPDR, non-proliferative diabetic retinopathy; TCNS, Toronto clinical neuropathy score; ROC, receiver operating characteristic

\section{Introduction}

Diabetes mellitus (DM) is considered as a global epidemic According to the International Diabetes Federation (IDF), DM affects more than 463 million people worldwide most of them are type 2 DM cases. The IDF estimates that the Middle East and North Africa (MENA) Region has the highest age adjusted prevalence of DM in adults in 2019, 2030 and 2045 (12.2\%, 13.3\% and 13.9\%, respectively). In 2019, the IDF ranked Saudi Arabia as the fourth highest country in the MENA region for the prevalence of diabetes in the age group 20-79 years with 4.3 million diagnosed with diabetes after Pakistan, Egypt, and Iran. ${ }^{1}$ Uncontrolled diabetes can cause many chronic macrovascular and microvascular complications. Long term diabetes complications may already be present in type 2 diabetics by the time they are appear soon after the onset of type 1 diabetes. Early detection and appropriate treatment are essential to prevent diabetes complications that may cause disability and death., ${ }^{2,3}$ Type 2 DM may cause microvascular complications such as retinopathy, nephropathy, and neuropathy. These microvascular complications are common and cause several degrees of visual impairment leading to blindness in retinopathy. ${ }^{1,4}$ Microvascular complications can cause proteinuria leading eventually to end-stage renal disease (ESRD) in nephropathy. ${ }^{5,6}$ Additionally, neuropathological complications can cause pain, numbness, and chronic recurrent infected ulcers in the extremities that may lead to amputation. ${ }^{7}$ Diabetic retinopathy is the leading cause of blindness within the working age population in most countries. ${ }^{8}$ In 2019, a systematic review indicated that the annual incidence of diabetic retinopathy is from $2.2 \%$ to $12.7 \%$ and annual progression to sight threatening diabetic retinopathy is from $3.4 \%$ to $12.3 \%{ }^{9}$ The World Health Organization (WHO) Universal Eye Health: A Global Action Plan 2014-2019 outlines the need to achieve a reduction in the prevalence of avoidable visual impairment and blindness including that related to diabetes, which is currently among the five most common causes of both moderate or severe visual impairment and blindness. WHO member states have committed by the year 2019 to reduce the prevalence of avoidable visual 
impairment by $25 \%$ compared to the baseline established by WHO in $2010 .{ }^{10}$ In Saudi Arabia, $10.8 \%$ of individuals with diabetes have diabetic nephropathy and $37 \%$ of them have ESRD. ${ }^{11,12}$ Furthermore, internationally among diabetics the prevalence of ESRD increases 10 fold compared with non-diabetics and almost $80 \%$ of ESRD is caused by $\mathrm{DM}$ and/or hypertension.

Primary prevention of diabetes is considered the most effective method to reduce the impact of diabetic kidney disease (DKD). Meanwhile, secondary prevention of DKD in those already diagnosed with diabetes can be achieved through early stage diagnoses and treatment of chronic kidney disease (CKD). ${ }^{1}$ Thus, albuminuria or glomerular filtration rate (GFR) screening is shown to be cost effective in diabetic and hypertensive patients. ${ }^{13}$ The most common form of diabetes related neuropathy is peripheral neuropathy. Distal nerves of the extremities are mainly affected, especially the feet. Patients usually experience pain and numbness due to the symmetrical sensory function alteration. ${ }^{1,7}$ The reported prevalence of diabetes related to peripheral neuropathy ranges from $16 \%$ to $87 \%$, with painful neuropathy reported in about $26 \%$ of diabetic adults. ${ }^{14,15}$ Moreover, lower limb amputation among diabetics increases 10 to 30 fold as compared to non-diabetics. ${ }^{16,17}$ Unfortunately, less than $30 \%$ of physicians can identify the signs of peripheral neuropathy caused by diabetes. ${ }^{18}$ As result of missed diagnoses, peripheral neuropathy causes high rates of morbidity and mortality. Thus, screening for retinopathy, nephropathy, and neuropathy is vital in the secondary prevention of diabetes microvascular complications. Therefore, having an annual screening for microvascular complications is recommended for diabetics, the screening for newly diagnosed type 2 diabetes is recommended at the time of diagnoses and for type 1 diabetes it is recommended 5 years after diagnoses. ${ }^{3}$ The main aim of the study is to test the effectiveness of a one stop screening clinic for retinopathy, nephropathy, and neuropathy for diabetic patients. In order to reach this aim, the first objective was to assess the feasibility of the one stop screening clinic. The second objective was to evaluate patient satisfaction. The third objective was to assess the diagnostic efficacy of two point of care devices which are DPN-Check and Sudoscan.

\section{Methods}

This research study was designed as a cross-sectional observational study. The study was done during a period of 3 months from February to March 2019. We used convenience sampling to select participants who attended the screening clinic of the Diabetes Care Center at King Salman Hospital, Riyadh, Saudi Arabia. A total of 260 diabetic patients participated in the study. The inclusion criteria for the study was diabetic patients aged 18 to 70 years. Participants known to have medical issues other than diabetes were excluded from the study, patients with co-morbidities such as chronic heart, renal or liver diseases. Patients with bilateral cataracts were also excluded from the study. An informed consent was signed by each participant. Participants had their microvascular screening done by physicians, optometrists and nurses in the one stop clinic. The protocol and point of care devices were supervised by an endocrinologist and a neurologist.

\section{Research tool}

The research tool used in this study was a self-administered online questionnaire in A well designed form was filled by the investigators, the form included some demographic questions such as age and gender. Other questions included the type and duration of diabetes. Vital signs such as blood pressure was measured using a digital sphygmomanometer. Digital retinal photography was used to assess retinopathy. Blood samples were taken from each participants for $\mathrm{HbA} 1 \mathrm{c}$ test and glomerular filtration rate (GFR), and urine samples were obtained to calculate albumin: creatinine ratio. Feet examination of participants for motor and sensory function was done using a tendon hammer, $128-\mathrm{Hz}$ tuning fork, and neurotip. Two point of care devices which are DPN-Check and Sudoscan were also used for feet examination. At the end participants were asked to complete a satisfaction survey for this one stop clinic. Definition of retinopathy is the presence of at least one micro-aneurysm, hemorrhage or exudates in either eye. We followed the International Council of Ophthalmology (ICO) recommendations for screening. ${ }^{19,20}$ According to the American Diabetes Association (ADA) recommendations for nephrology screening, patients were considered to have normal albuminuria if the albumin: creatinine ratio was $<30 \mathrm{mg} / \mathrm{g}$, microalbuminuria if the ratio was $30-300 \mathrm{mg} / \mathrm{g}$, and macro-albuminuria if the ratio was $>300 \mathrm{mg} / \mathrm{g} .{ }^{3,21}$ Staging of CKD was according to the guidelines of the National Kidney Foundation, and defined as stage 1 if GFR $\geq 90 \mathrm{~mL} / \mathrm{min} / 1.73 \mathrm{~m}^{2}$, stage 2 if GFR 60 to $89 \mathrm{~mL} / \mathrm{min} / 1.73$ $\mathrm{m}^{2}$, stage 3 if GFR 30 to $59 \mathrm{~mL} / \mathrm{min} / 1.73 \mathrm{~m}^{2}$, stage 4 if GFR 15 to $29 \mathrm{~mL} / \mathrm{min} / 1.73 \mathrm{~m}^{2}$, and stage 5 if GFR $<15 \mathrm{~mL} / \mathrm{min} / 1.73 \mathrm{~m}^{2}{ }^{22}$ Feet examination was done as per Boulton. Painful neuropathic symptoms were considered positive if the neuropathy symptom score (NSS) was $\geq 5$. The neuropathy disability score (NDS) determined the presence of diabetic peripheral neuropathy (DPN), DPN was considered present if the NDS $\geq 3$. The NDS results were considered as the gold standard to be compared with the results of DPN-Check and Sudoscan. We divided the participants into four groups which are no DPN, mild DPN, moderate DPN, and severe DPN. No DPN if NDS $<3$, mild DPN if NDS is $3-5$, moderate DPN if NDS is 6-8, and severe DPN if NDS $>8 .{ }^{23,24}$ DPN-Check is a point of care device developed by Neurometrix. The device measures the sural sensory nerve conduction velocity $(\mathrm{SNCV} ; \mathrm{m} / \mathrm{s}$ ) and the sural nerve action potential (SNAP; $\mu \mathrm{V})$. The DPN- Check is a hand held device with a screen to display the SNCV and the SNAP. For the diagnoses of DPN using DPNCheck, we had to have SNCV $<40 \mathrm{~m} / \mathrm{s}$ and/or SNAP $<4 \mu \mathrm{V}$ for both feet. ${ }^{25,26}$ Sudoscan is a point of care device developed by Impeto Medical. The device provides assessment of sudomotor function. Sudoscan measures voltage electrochemical skin conductance (ESC; $\mu \mathrm{S}$ ) for both hands and feet of the participants. For the diagnoses of DPN using Sudoscan the ESC for both feet had to be less than $60 \mu \mathrm{S.}^{27}$

\section{Statistical analysis}

The data is presented as either mean standard deviation or percentage. Independent T-test was used to calculate the variances between variable. Logistic regression analysis was done to calculate the odds ratio of having DPN according to SNCV and SNAP. For Sudomotor function to detect DPN, receiver operating characteristic curve (ROC) analysis, sensitivity and specificity were used to assess SNCV and SNAP. A p- value of $<0.05$ was used to report the statistical significance. Data was analyzed using SPSS 25.0.

\section{Results}

The study included 260 participants, Table 1 shows that around $39 \%$ of participants were male participants and almost $61 \%$ were female. The mean age of the participants was 51 years. Most of the participants were type 2 diabetic patient (93.5\%), and only $6.5 \%$ of them were type 1 diabetic patient. The patients' acceptance and satisfaction rates of the one stop clinic were $100 \%$. Table 2 shows the association between the participants in the four groups (no DPN, mild DPN, moderate DPN, and severe DPN) to mean age, HbAlc, systolic 
and diastolic blood pressure, and albumin to creatinine ratio. The table also shows the association between the participants in the four groups to participants with type 2 diabetes and positive monofilament test. Finally, the table shows the mean SNCV and SNAP according to DPN-Check and mean ESC according to Sudoscan in each of the four groups of participants. Retinopathy screening showed the prevalence of non-proliferative diabetic retinopathy (NPDR) to be $11 \%$. Meanwhile, the prevalence of maculopathy was $1.5 \%$. Regarding nephrology screening, the prevalence of micro-albuminuria was $18.6 \%$ and macro-albuminuria was $1.9 \%$. Also, the prevalence of chronic kidney disease (CKD) stage 3 was $4.2 \%$ and stage 4 was $0.4 \%$ among participants in the study. While screening for neuropathy, we found that neuropathic symptoms were present in $40.7 \%$ of the participants. The prevalence of DNP according to NDS, which is our gold standard test was $13.8 \%$ and using $10-\mathrm{g}$ monofilament test was $7.69 \%$. Meanwhile, the prevalence of DNP according to DPN-check was $40.9 \%$, and according to Sudoscan was $73 \%$. The DPN-check showed to have sensitivity of $62.9 \%$ and specificity of $69 \%$, area under the ROC curve 0.70 Figure 1. The Sudoscan showed to have sensitivity of $29.4 \%$ and specificity of $85.7 \%$, area under the ROC curve 0.67 Figure 1.

Table I Personal characteristics of the participants $(n=260)$

\begin{tabular}{ll}
\hline Number of participants & 260 \\
\hline Mean (SD) age, years & $51.2(\mathrm{I}$ I.7) \\
Gender & $102(39.2 \%)$ \\
Male, n (\%) & I58 (60.7\%) \\
Female, n (\%) & $243(93.5 \%)$ \\
Type 2 diabetes, n (\%) & $17(6.5 \%)$ \\
\hline
\end{tabular}

Table 2 Clinical characteristics of the participants $(n=260)$

\begin{tabular}{|c|c|c|c|c|}
\hline & No DPN & Mild DPN & Moderate DPN & Severe DPN \\
\hline Number of participants, n (\%) & $224(86.2)$ & $28(10.8)$ & $5(1.9)$ & $3(1.2)$ \\
\hline Mean (SD) age, years & $51.03(12.3)$ & $56.6(7.08)$ & $46.2(10.4)$ & $53.6(7.09)$ \\
\hline Type 2 diabetes, $\mathrm{n}(\%)$ & 209 ( 93.3$)$ & $27(96)$ & $5(100)$ & $2(67)$ \\
\hline Mean (SD) HbAlc \% & $8.59(2.1)$ & $9.2(2.2)$ & $8.56(0.89)$ & $8.93(2.29)$ \\
\hline Mean systolic blood pressure, $\mathrm{mmHg}(\mathrm{SD})$ & I35.7 ( 2 |.5 ) & | 38.7 ( 25.2$)$ & $154(46.6)$ & $134(19.8)$ \\
\hline Mean diastolic blood pressure, $\mathrm{mmHg}(\mathrm{SD})$ & $81.58(9.5)$ & $76(9.6)$ & $86.3(30)$ & $87(15.5)$ \\
\hline Mean (SD) albumin:creatini ne ratio & $34.4(97)$ & $25.5(24.5)$ & $73.5(120)$ & $8.26(3.9)$ \\
\hline Positive I0-g monofilament test, n (\%) & $8(3.6)$ & $8(28.6)$ & $3(60)$ & I ( 33 ) \\
\hline \multicolumn{5}{|l|}{ DPN-Check } \\
\hline Mean (SD) right sural SNCV, m/s & $44.8(18.4)$ & $32.5(24.4)$ & $48(10.65)$ & $18(31.2)$ \\
\hline Mean (SD) right sural, SNAP, $\mu \mathrm{V}$ & $7.2(5.5)$ & $3.03(2.5)$ & $7.8(5.36)$ & $2(3.5)$ \\
\hline Mean (SD) left sural SNCV, m/s & $46.37(17.17)$ & $34.3(22.9)$ & $34.2(23.6)$ & $18(31.2)$ \\
\hline Mean (SD) left sural SNAP, $\mu \mathrm{V}$ & $7.34(5.7)$ & $3.25(2.59)$ & $3(2.23)$ & $3.33(3.51)$ \\
\hline \multicolumn{5}{|l|}{ Sudoscan } \\
\hline Mean (SD) hand ESC, $\mu S$ & $65.5(16.6)$ & $52(21.7)$ & $60.8(16.9)$ & $73.66(5.77)$ \\
\hline Mean (SD) foot & $56.9(18.57)$ & $39.29(21.3)$ & $62.2(11.36)$ & $56.33(3.51)$ \\
\hline ESC, & & & & \\
\hline
\end{tabular}




\section{ROC Curve}

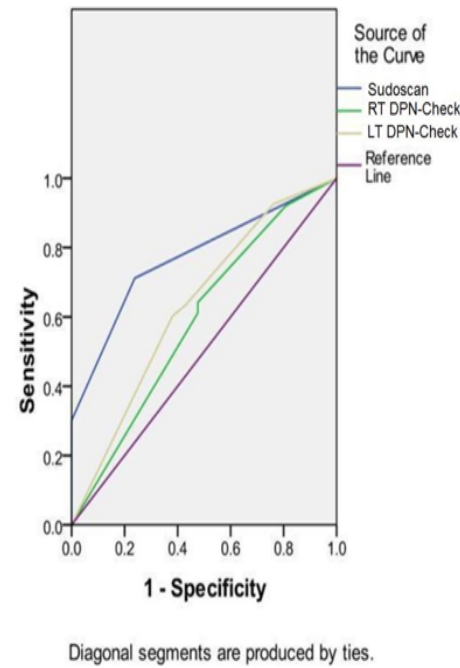

Figure I DPN-check and sudoscan ROC curve.

\section{Discussion}

Studies found that the barriers for microvascular screening in diabetic patients were difficulties in getting appointments, lengthy waiting periods, lack of knowledge about the importance of screening especially in the asymptomatic phase, and the insufficient coordination between physicians and screeners. ${ }^{28,29}$ This made health care providers suggested reform to overcome these barriers. ${ }^{30}$ Therefore, integrating the ophthalmology, nephrology, and neurology clinics into one clinic provides affordability, accessibility, and efficiency to insure improvement in the quality of care and prevention from microvascular complications. In the present study, patients' acceptance and satisfaction rates were $100 \%$, comparing participants' satisfaction of our study with a similar study done in the UK that found that $91.1 \%$ of participants favored the one stop screening clinic. ${ }^{31}$ It is clear that the one stop screening clinic saved time and effort for patients, instead of following up with 3 different clinics for the microvascular screening. In the present research, the prevalence of NPDR among participants was similar to some studies done in Saudi Arabia. ${ }^{32,33}$ Other studies in Saudi Arabia reported a higher prevalence rate of NPDR, but all studied emphasized on the importance of retinopathy referral and screening. ${ }^{34,35}$ Maculopathy prevalence rate among diabetic patients in this research was $1.5 \%$, which correlated with another study that was done in Abha, Saudi Arabia that reported maculopathy prevalence of $2.5 \% .^{32}$ These relatively high prevalence rates show the importance of retinopathy screening for diabetics in Saudi Arabia. Thus, the one stop screening clinic can help overcome referral concerns addressed in previous literature. ${ }^{34}$ Our study found the prevalence of CKD stage 3 was $4.2 \%$ and stage 4 was $0.4 \%$, this prevalence rate was similar with the prevalence of CKD in the general population in Saudi Arabia and some other countries. ${ }^{36,37}$ Another Dutch study that included showed that CKD stage 3 among diabetic participants was $17.1 \%$ and CKD stage 4 was $0.4 \%$, the difference in the CKD stage 3 prevalence might be because the Dutch study included patients above the age of $25 .{ }^{38}$ Micro-albuminuria and macro-albuminuria prevalence rates in our study also matched the results reported in a couple of studies with a similar population sample. ${ }^{39,40} \mathrm{CKD}$ prevalence rates in Saudi Arabia is correlated with other countries in different parts of the world, which indicate the significance of following the international guidelines in screening for CKD. In agreement with a similar recent study done in the UK, the 10-g monofilament test underestimated the prevalence of DPN as compared to the gold standard test. ${ }^{31}$ However, our study showed that non- invasive point of care devices such as DPN-check and Sudoscan overestimated the prevalence of DPN compared to our gold standard test. On the other hand, the UK study showed that both DPN-check and Sudoscan prevalence rates of DPN matched their gold standard test results. ${ }^{31}$ The UK study used Toronto Clinical Neuropathy Score (TCNS) as their gold standard test, the TCNS reliability and validity perfectly correlate with our gold standard test the NDS. ${ }^{41}$ Other studies showed different sensitivity and specificity for both DPN-check and Sudoscan than what we found in our study. ${ }^{25,27,31}$ We believe that more research is needed to clarify the sensitivity and specificity of DPN-check and Sudoscan.

\section{Conclusion}

It is feasible to have a one stop clinic service that combines retinopathy, nephropathy, and neuropathy screening. The one stop screening clinic showed prevalence rates of retinopathy, nephropathy, and neuropathy similar to those found in other studies done nationally and internationally. Thus, the one stop clinic approach can detect microvascular disease, it is highly accepted, and reduces clinical visits. Therefore, applying a one stop microvascular screening clinic in the diabetes care centers in Saudi Arabia could be very effective for early diagnosis of diabetes microvascular complications. Finally, more research should study the cost effectiveness of using noninvasive point of care devices for assessing both large and small nerve fibers for diagnosing DPN.

\section{Acknowledgments}

None.

\section{Conflicts of interest}

The authors declare that there are no conflicts of interest.

\section{Funding}

None.

\section{References}

1. Atlas D. International diabetes federation. IDF diabetes Atlas, 9th edn. Brussels, Belgium: International diabetes federation, 2019.

2. Girach A, Manner D, Porta M. Diabetic microvascular complications: can patients at risk be identified? a review. Int J Clin Pract. 2006;60(11):14711483 .

3. ADA. 11. Microvascular Complications and foot care: standards of medical care in diabetes-2019. Diabetes Care. 2019;42(Suppl 1):S124 S138.

4. Viswanath K, McGavin DD. Diabetic retinopathy: clinical findings and management. Community Eye Health. 2003;16(46):21-24.

5. Gross JL, de Azevedo MJ, Silveiro SP, et al. Diabetic nephropathy: diagnosis, prevention, and treatment. Diabetes Care. 2005;28(1):164176.

6. King H, Aubert RE, Herman WH. Global burden of diabetes, 19952025: prevalence, numerical estimates, and projections. Diabetes Care. 1998;21(9):1414-1431.

7. Pop-Busui R, Boulton AJ, Feldman EL, et al. Diabetic neuropathy: a position statement by the American diabetes association. Diabetes Care. 2017;40(1):136-154. 
8. Solomon SD, Chew E, Duh EJ, et al. Diabetic retinopathy: a position statement by the American diabetes association. Diabetes Care. 2017;40(3):412-418.

9. Sabanayagam C, Banu R, Chee ML, et al. Incidence and progression of diabetic retinopathy: a systematic review. Lancet Diabetes Endocrinol. 2019;7(2):140-149.

10. Gupta N, Kocur I. Chronic eye disease and the WHO universal eye health global action plan 2014-2019. Can J Ophthalmol. 2014;49(5):403-405.

11. Al-Rubeaan K, Youssef AM, Subhani SN, et al. Diabetic nephropathy and its risk factors in a society with a type 2 diabetes epidemic: a Saudi national diabetes registry-based study. PLoS One. 2014;9(2):e88956.

12. Al-Rubeaan K, Siddiqui K, Alghonaim M, et al. The Saudi diabetic kidney disease study (Saudi-DKD): clinical characteristics and biochemical parameters. Ann Saudi Med. 2018;38(1):46-56.

13. Komenda P, Ferguson TW, Macdonald K, et al. Cost-effectiveness of primary screening for CKD: a systematic review. Am J Kidney Dis. 2014;63(5):789-797.

14. Sobhani S, Asayesh H, Sharifi F, et al. Prevalence of diabetic peripheral neuropathy in Iran: a systematic review and meta-analysis. $J$ Diabetes Metab Disord. 2014;13(1):97.

15. Davies M, Brophy S, Williams R, et al. The prevalence, severity, and impact of painful diabetic peripheral neuropathy in type 2 diabetes. Diabetes Care. 2006;29(7):1518-1522.

16. Siitonen OI, Niskanen LK, Laakso M, et al. Lower-extremity amputations in diabetic and nondiabetic patients. a population-based study in eastern Finland. Diabetes Care. 1993;16(1):16-20.

17. Trautner C, Haastert B, Giani G, et al. Incidence of lower limb amputations and diabetes. Diabetes Care. 1996;19(9):1006-1009.

18. Griffith RJ. Williams textbook of endocrinology, $10^{\text {th }}$ edition: P Reed Larsen, Henry M Kronenberg, Shlomo Melmed, et al. Philadelphia, W.B. Saunders Company, 2003, 1927 pp. Journal of Pediatric and Adolescent Gynecology. 2004;17(3):217-218.

19. Aiello LP, Gardner TW, King GL, et al. Diabetic retinopathy. Diabetes Care. 1998;21(1):143-156.

20. Wong TY, Sun J, Kawasaki R, et al. Guidelines on diabetic eye care: the international council of ophthalmology recommendations for screening, follow-up, referral, and treatment based on resource settings. Ophthalmology. 2018;125(10):1608-1622.

21. Basi S, Fesler P, Mimran A, et al. Microalbuminuria in type 2 diabetes and hypertension: a marker, treatment target, or innocent bystander? Diabetes Care. 2008;31 Suppl 2:S194-S201.

22. NKF. K/DOQI clinical practice guidelines for chronic kidney disease: evaluation, classification, and stratification. Am J Kidney Dis. 2002;39(2 Suppl 1):S1-S266.

23. Boulton AJ, Armstrong DG, Albert SF, et al. Comprehensive foot examination and risk assessment: a report of the task force of the foot care interest group of the American diabetes association, with endorsement by the American association of clinical endocrinologists. Diabetes Care. 2008;31(8):1679-1685.

24. Young MJ, Boulton AJ, MacLeod AF, et al. A multicentre study of the prevalence of diabetic peripheral neuropathy in the United Kingdom hospital clinic population. Diabetologia. 1993;36(2):150-154.
25. Lee JA, Halpern EM, Lovblom LE, et al. Reliability and validity of a point- of-care sural nerve conduction device for identification of diabetic neuropathy. PLoS One. 2014;9(1):e86515.

26. Kong X, Schoenfeld DA, Lesser EA, et al. Implementation and evaluation of a statistical framework for nerve conduction study reference range calculation. Comput Methods Programs Biomed. 2010;97(1):1-10.

27. Casellini CM, Parson HK, Richardson MS, et al. Sudoscan, a noninvasive tool for detecting diabetic small fiber neuropathy and autonomic dysfunction. Diabetes Technol Ther. 2013;15(11):948-953.

28. Piyasena M, Murthy GVS, Yip JLY, et al. Systematic review on barriers and enablers for access to diabetic retinopathy screening services in different income settings. PLoS One. 2019;14(4):e0198979.

29. Neale EP, Middleton J, Lambert K. Barriers and enablers to detection and management of chronic kidney disease in primary healthcare: a systematic review. BMC Nephrol. 2020;21(1):83.

30. Chin $\mathrm{MH}$, Cook S, Jin L, et al. Barriers to providing diabetes care in community health centers. Diabetes Care. 2001;24(2):268-274.

31. Binns-Hall O, Selvarajah D, Sanger D, et al. One-stop microvascular screening service: an effective model for the early detection of diabetic peripheral neuropathy and the high-risk foot. Diabet Med. 2018;35(7):887-894.

32. Ahmed RA, Khalil SN, Al-Qahtani MA. Diabetic retinopathy and the associated risk factors in diabetes type 2 patients in Abha, Saudi Arabia. $J$ Family Community Med. 2016;23(1):18-24.

33. Al-Khaldi YM, Khan MY, Khairallah SH. Audit of referral of diabetic patients. Saudi Med J. 2002;23(2):177-181.

34. Khan AR, Wiseberg JA, Lateef ZA, et al. Prevalence and determinants of diabetic retinopathy in Al hasa region of Saudi Arabia: primary health care centre based cross-sectional survey, 2007-2009. Middle East Afr J Ophthalmol. 2010;17(3):257-263.

35. Al Ghamdi AH, Rabiu M, Hajar S, et al. Rapid assessment of avoidable blindness and diabetic retinopathy in Taif, Saudi Arabia. Br J Ophthalmol. 2012;96(9):1168-1172.

36. Ahmed HG, Ginawi IA, Al-Hazimi AM. Prevalence estimates of chronic kidney disease in hail region, KSA: in a comprehensive Survey. International Journal of Science and Research. 2014;3(7):252-256.

37. Duan JY, Duan GC, Wang CJ, et al. Prevalence and risk factors of chronic kidney disease and diabetic kidney disease in a central Chinese urban population: a cross- sectional survey. BMC Nephrol. 2020;21(1):115.

38. van der Meer V, Wielders HP, Grootendorst DC, et al. Chronic kidney disease in patients with diabetes mellitus type 2 or hypertension in general practice. Br J Gen Pract. 2010;60(581):884-890.

39. Lee ES, Tang WE. The prevalence of albuminuria among diabetic patients in a primary care setting in Singapore. Singapore Med J. 2015;56(12):681686.

40. Stephenson JM, Fuller JH. Microalbuminuria is not rare before 5 years of IDDM. EURODIAB IDDM complications study group and the WHO multinational study of vascular disease in diabetes Study Group. $J$ Diabetes Complications. 1994;8(3):166-173.

41. Bril V, Tomioka S, Buchanan RA, et al. Reliability and validity of the modified Toronto clinical neuropathy score in diabetic sensorimotor polyneuropathy. Diabet Med. 2009;26(3):240-246. 